\title{
Equation of State for Nucleonic Matter and its Quark Mass Dependence from the Nuclear Force in Lattice QCD
}

\author{
Takashi Inoue ${ }^{1}$, Sinya Aoki ${ }^{2,3}$, Takumi Doi $^{4}$, Tetsuo Hatsuda ${ }^{4,5}$, \\ Yoichi Ikeda ${ }^{4}$, Noriyoshi Ishii ${ }^{3}$, Keiko Murano ${ }^{4}$, Hidekatsu Nemura ${ }^{3}$, Kenji Sasaki ${ }^{3}$ \\ (HAL QCD Collaboration) \\ ${ }^{1}$ Nihon University, College of Bioresource Sciences, Kanagawa 252-0880, Japan \\ ${ }^{2}$ Yukawa Institute for Theoretical Physics, Kyoto University, Kyoto 606-8502, Japan \\ ${ }^{3}$ Center for Computational Sciences, University of Tsukuba, Ibaraki 305-8571, Japan \\ ${ }^{4}$ Theoretical Research Division, Nishina Center, RIKEN, Saitama 351-0198, Japan \\ ${ }^{5}$ Kavli IPMU (WPI), The University of Tokyo, Chiba 277-8583, Japan
}

\begin{abstract}
Quark mass dependence of the equation of state (EOS) for nucleonic matter is investigated, on the basis of the Brueckner-Hartree-Fock method with the nucleon-nucleon interaction extracted from lattice QCD simulations. We observe saturation of nuclear matter at the lightest available quark mass corresponding to the pseudoscalar meson mass $\simeq 469 \mathrm{MeV}$. Mass-radius relation of the neutron stars is also studied with the EOS for neutron-star matter from the same nuclear force in lattice QCD. We observe that the EOS becomes stiffer and thus the maximum mass of neutron star increases as the quark mass decreases toward the physical point.
\end{abstract}

PACS numbers: 12.38.Gc, 13.75.Cs, 21.65.Mn, 26.60.Kp

The equation of state (EOS) for hadronic matter is a key quantity for understanding the physics of compact stars and explosive phenomena in astrophysics. From the observational point of view, recent reports on massive neutron stars put stringent constraints on the EOS [1]. In the future, neutrinos from core-collapsed supernovae and gravitational waves from neutron star mergers will give further constraints on the EOS [2, 3]. From the theoretical point of view, various approaches to calculate the EOS have been pursued so far, e.g. the BruecknerBethe-Goldstone theory [4], the quantum Monte Carlo simulations [5], and the chiral effective theories [6, 7]. Although they provide reasonable descriptions of the nuclear matter at low density, it is still beyond the scope of these approaches to answer the fundamental questions such as the quark-mass $\left(m_{q}\right)$ dependence of the nuclear saturation property and the maximum mass of neutron stars. These questions can only be answered by manybody techniques with hadronic interactions obtained by lattice QCD simulations for different $m_{q}$.

The purpose of this Letter is to make a first exploratory study for the nuclear and neutron matter EOS by combining the Brueckner-Hartree-Fock (BHF) many-body theory with the nuclear force obtained from lattice QCD simulations. In particular we study how the saturation develops in nuclear matter and how the mass-radius relation of the neutron star changes as a function of $m_{q}$ : Such $m_{q}$ dependence of the EOS gives us useful information on the physics of strongly interacting nucleons, even though the values of $m_{q}$ in this study are still away from the physical one. In addition, it is certainly important to establish a direct connection between lattice QCD and the physics of the nucleonic matter.

The nuclear force which we adopt in this Letter is taken from the zero-strangeness sector of the octet-
TABLE I: $M_{\mathrm{PS}}, M_{\mathrm{V}}$ and $M_{\mathrm{B}}$ denote hadron masses corresponding to the pseudoscalar meson, vector meson and octet baryon, respectively, with the $S U(3)$ symmetric hopping parameter $\kappa_{u d s}$ [9]. Trajectory length $N_{\text {traj }}$ and number of configurations $N_{\text {cfg }}$ are also shown.

\begin{tabular}{c|r|r|c|c}
\hline \hline$\kappa_{u d s}$ & \multicolumn{1}{|c|}{$M_{\mathrm{PS}}[\mathrm{MeV}]$} & $M_{\mathrm{V}}[\mathrm{MeV}]$ & $M_{\mathrm{B}}[\mathrm{MeV}]$ & $N_{\text {cfg }} / N_{\text {traj }}$ \\
\hline 0.13660 & $1170.9(7)$ & $1510.4(0.9)$ & $2274(2)$ & $420 / 4200$ \\
0.13710 & $1015.2(6)$ & $1360.6(1.1)$ & $2031(2)$ & $360 / 3600$ \\
0.13760 & $836.5(5)$ & $1188.9(0.9)$ & $1749(1)$ & $480 / 4800$ \\
0.13800 & $672.3(6)$ & $1027.6(1.0)$ & $1484(2)$ & $360 / 3600$ \\
0.13840 & $468.6(7)$ & $829.2(1.5)$ & $1161(2)$ & $720 / 3600$ \\
\hline \hline
\end{tabular}

baryon potentials in the flavor-SU(3) limit calculated on the lattice [8, 9], where the renormalization group improved Iwasaki gauge action and the nonperturbatively improved Wilson quark action were employed on a $32^{3} \times 32$ lattice with the lattice spacing $a=0.121(2)$ $\mathrm{fm}$. The potentials were derived from the imaginary-time Nambu-Bethe-Salpeter wave functions by the HAL QCD method [10 12], at the quark masses corresponding to the pseudoscalar meson masses $\left(M_{\mathrm{PS}}\right)$ ranging between 469 and $1171 \mathrm{MeV}$. Shown together in Table I are the vector meson mass $\left(M_{\mathrm{V}}\right)$ and the baryon mass $\left(M_{\mathrm{B}}\right)$ for these quark masses.

In Fig. 1 we show the $N N$ potentials obtained from fits to the lattice data in $\mathrm{S}$ and $\mathrm{D}$-waves at $M_{\mathrm{PS}} \simeq 469$ $\mathrm{MeV}$. These potentials share common features with phenomenological potentials, i.e., a strong repulsive core at short distance and the attractive pocket at intermediate distance, so that the ${ }^{1} \mathrm{~S}_{0}$ phase shift in Fig. 2 shows qualitatively similar behavior with the experimental data [9]. While the phase shift in the ${ }^{3} \mathrm{~S}_{1}$ channel shows stronger attraction at low energies than that of the 


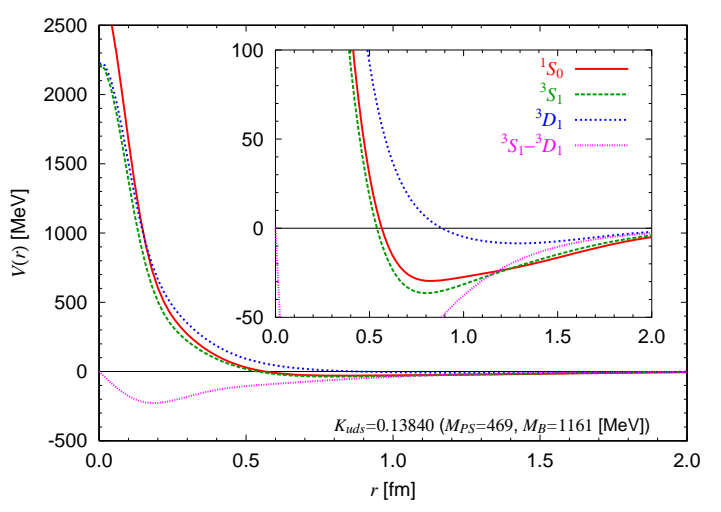

FIG. 1: The $N N$ potentials for $\mathrm{S}$ and $\mathrm{D}$ waves extracted from lattice $\mathrm{QCD}$ data at $M_{\mathrm{PS}}=469 \mathrm{MeV}$ in the flavor- $S U(3)$ limit. The lines are obtained by the least $\chi^{2}$ fit to the lattice data (see [9] for the fit function).

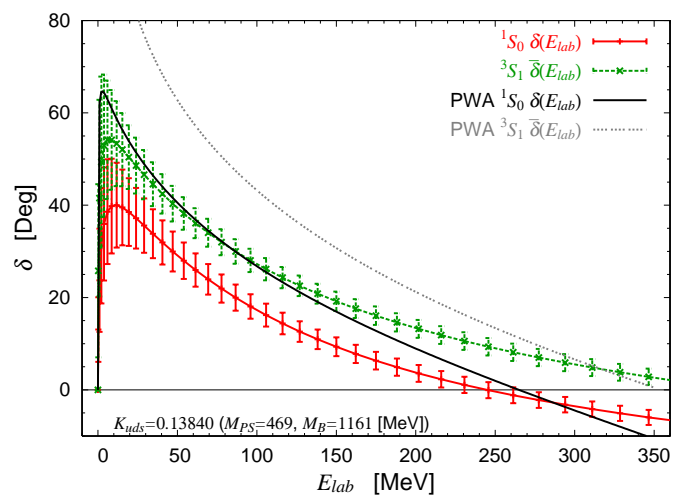

FIG. 2: Phases shifts of $N N$ scattering as a function of energy in the laboratory frame, extracted from lattice QCD data at $M_{\mathrm{PS}}=469 \mathrm{MeV}$ in the flavor-SU(3) limit: The black and gray dashed lines are partial wave analysis (PWA) of experimental data taken from NN-OnLine (http://nn-online.org/) .

${ }^{1} \mathrm{~S}_{0}$ channel due to ${ }^{3} \mathrm{~S}_{1^{-}}{ }^{3} \mathrm{D}_{1}$ mixing, it is still insufficient to form the deuteron bound state even at $M_{\mathrm{PS}} \simeq 469$ $\mathrm{MeV}$ [9, 13]. Although we found no bound state in two and three nucleon systems, there exists a four-nucleon bound state, namely, ${ }^{4} \mathrm{He}$, with about $5 \mathrm{MeV}$ binding energy at $M_{\mathrm{PS}} \simeq 469 \mathrm{MeV}[9]$.

Let us now study the EOS for nuclear matter and neutron matter in the leading order of the BruecknerBethe-Goldstone (BBG) hole-line expansion, i.e., the Brueckner-Hartree-Fock (BHF) theory (see e.g. [4]), where the total ground-state energy $E$ at zero temperature with the nucleon mass $M_{N}$ and the Fermi momentum $k_{F}$ reads

$$
E=\sum_{k}^{k_{F}} \frac{k^{2}}{2 M_{N}}+\frac{1}{2} \sum_{k, k^{\prime}}^{k_{F}} \operatorname{Re}\left\langle k k^{\prime}\left|G\left(e(k)+e\left(k^{\prime}\right)\right)\right| k k^{\prime}\right\rangle_{A}
$$

with $\left|k k^{\prime}\right\rangle_{A} \equiv\left|k k^{\prime}\right\rangle-\left|k^{\prime} k\right\rangle$. To simplify the notation, spin and isospin indices of the nucleons are included in

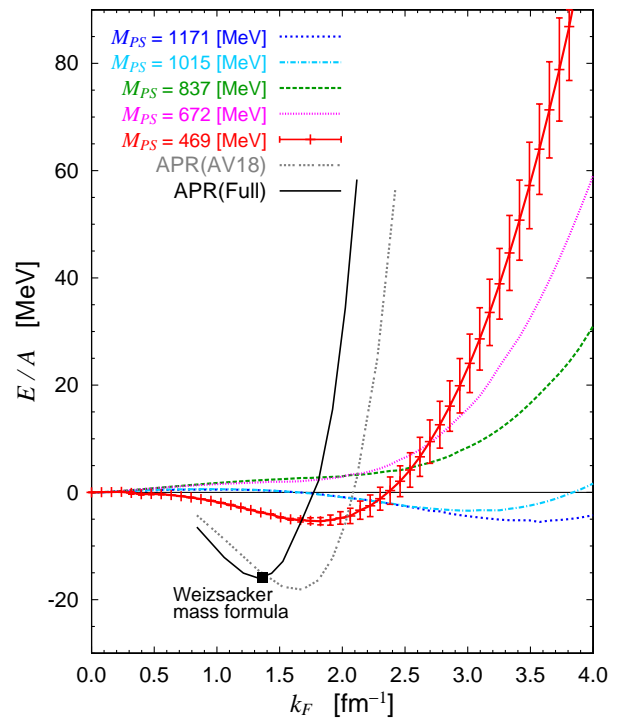

FIG. 3: Ground state energy per nucleon $(E / A)$ for symmetric nuclear matter as a function of $k_{F}$ obtained by the BHF theory with the $N N$ potential from lattice QCD. Filled square indicates the empirical saturation point, and the curves labeled APR are taken from Ref. [16] with and without the phenomenological three nucleon force. The error bars for the result at $M_{\mathrm{PS}} \simeq 469 \mathrm{MeV}$ are the statistical uncertainties estimated by dividing the gauge configurations into two sets.

the label $k$. The $G$ matrix, describing the in-medium effective interaction of the two nucleons, obeys the BetheGoldstone integral equation with the bare interaction $V$,

$$
\begin{aligned}
& \left\langle k_{1} k_{2}|G(\omega)| k_{3} k_{4}\right\rangle=\left\langle k_{1} k_{2}|V| k_{3} k_{4}\right\rangle \\
& \quad+\sum_{k_{5}, k_{6}} \frac{\left\langle k_{1} k_{2}|V| k_{5} k_{6}\right\rangle Q\left(k_{5}, k_{6}\right)\left\langle k_{5} k_{6}|G(\omega)| k_{3} k_{4}\right\rangle}{\omega-e\left(k_{5}\right)-e\left(k_{6}\right)}
\end{aligned}
$$

where $Q\left(k, k^{\prime}\right)=\theta\left(k-k_{F}\right) \theta\left(k^{\prime}-k_{F}\right)$ is the Pauli exclusion operator tp prevent two nucleons from scatterng into occupied states. The single particle energy $e(k)=$ $\frac{k^{2}}{2 M_{N}}+U(k)$ contains the single-particle potential $U(k)$ defined by

$$
U(k)=\sum_{k^{\prime} \leq k_{F}} \operatorname{Re}\left\langle k k^{\prime}\left|G\left(e(k)+e\left(k^{\prime}\right)\right)\right| k k^{\prime}\right\rangle_{A} .
$$

The $G$ matrix is obtained by solving Eqs.(2) 3) selfconsistently, using the lattice QCD $N N$ potential for $V$ and the lattice QCD nucleon mass for $M_{N}$. Then the total energy $E$ is obtained from Eq.(11). We employ the matrix inversion method [14] with the continuous choice for $U\left(k>k_{F}\right)$ [15]. Because of the limitation for the lattice QCD potentials available at present, we keep the partial-wave decomposition of the $G$-matrix only up to ${ }^{1} \mathrm{~S}_{0},{ }^{3} \mathrm{~S}_{1}$, and ${ }^{3} \mathrm{D}_{1}$ channels.

Figure. 3 shows the ground state energy per nucleon $(E / A)$ for the symmetric nuclear matter $(Z=N=A / 2$ with the proton number $Z$, neutron number $N$ and the 


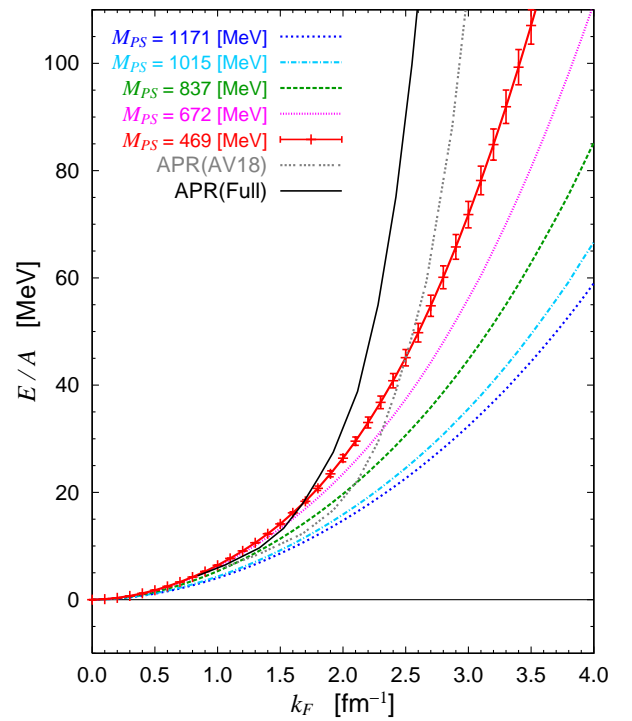

FIG. 4: Ground state energy per neutron for the pure neutron matter as a function of the Fermi momentum. Details of the calculation are the same as Fig. 3

mass number $A=N+Z$ ) as a function of $k_{F}$ for different $m_{q}$. The most important feature of the symmetric nuclear matter in the real world is its saturation property; i.e., $E / A$ takes the minimum at normal nuclear matter density $\rho_{0}$. The empirical saturation point from the Weizsäcker mass formula corresponds to $\left(k_{F}, E / A\right) \simeq\left(1.36 \mathrm{fm}^{-1},-15.7 \mathrm{MeV}\right)$ as indicated in Fig. 3. Also, we show the results of Ref. [16] which employs the variational method with AV18 $N N$ potential with and without phenomenological three-nucleon $N N N$ force.

Our result at the lightest quark mass $\left(M_{\mathrm{PS}} \simeq 469\right.$ $\mathrm{MeV}$ ) in Fig. 3indicates that the symmetric nuclear matter becomes a self-bound system with a saturation point $\left(k_{F}, E / A\right) \simeq\left(1.83 \pm 0.16 \mathrm{fm}^{-1},-5.4 \pm 0.5 \mathrm{MeV}\right)$. Here the errors are statistical uncertainties estimated by dividing the gauge configurations into two sets. This is the first time that the nuclear force obtained from first principle lattice QCD simulations leads to the saturation in the symmetric nuclear matter. The saturation point, however, deviates from the empirical point primarily due to heavy quark masses in our lattice simulation and the lack of $N N N$ force in our BHF calculation.

Also, we find nontrivial $m_{q}$ dependence of the EOS: the saturation disappears at intermediate quark masses $\left(M_{\mathrm{PS}} \simeq 672,837 \mathrm{MeV}\right)$ and appears again at the heavy quark mass region $\left(M_{\mathrm{PS}} \simeq 1015,1171 \mathrm{MeV}\right)$. This implies that the saturation originates from a subtle balance between short-range repulsion and the intermediate attraction of the nuclear force which have different $m_{q}$ dependence [9]. The saturation points for $M_{\mathrm{PS}} \simeq 1015,1171$ $\mathrm{MeV}$ locate at more than $10 \rho_{0}$, so that the effect of finite lattice spacing and the validity of BHF theory need to

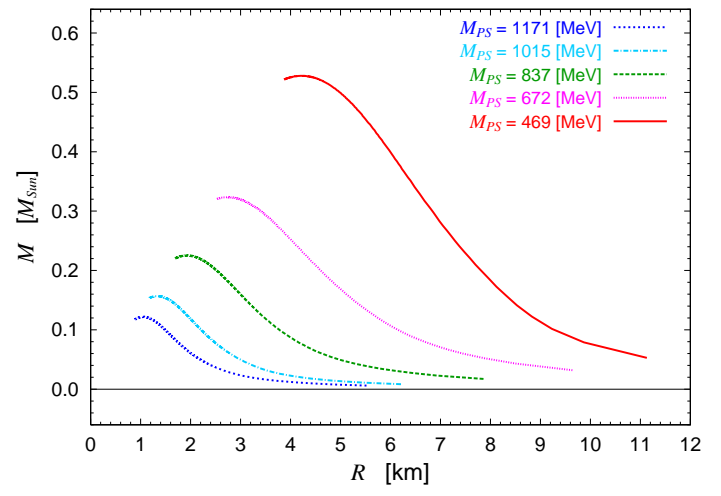

FIG. 5: Mass-radius relation of the neutron star. Neutronstar matter consists of $n, p, e^{-}$, and $\mu^{-}$with charge neutrality and chemical equilibrium. EOS for the nucleons is obtained by an interpolation between Fig. 3. and Fig. 4 under the parabolic approximation.

be carefully checked for those cases. Our restriction of the $N N$ interactions and the $G$ matrix to the ${ }^{1} \mathrm{~S}_{0}$ and ${ }^{3} \mathrm{~S}_{1}-{ }^{3} \mathrm{D}_{1}$ channels may not be a bad approximation, since the $S$ wave is known to be a dominant contribution and higher partial waves tend to cancel with each other near the physical saturation point 17. Nevertheless, we will include the results of on-going spin-orbit force calculation on the lattice [18] in the near future. In addition, we need to include the three-nucleon force from lattice QCD simulations [19] to make the EOS at high density more realistic.

Figure. 4]shows $E / A$ for the pure neutron matter $(N=$ $A)$ as a function of $k_{F}$. In this case, neutron matter is not self-bound due to large Fermi energy. The pressure of the system is proportional to the slope of $E / A$ as $P=$ $\rho^{2} \frac{\partial(E / A)}{\partial \rho}=\frac{k_{F}^{4}}{9 \pi^{2}} \frac{\partial(E / A)}{\partial k_{F}} ;$ Fig. 团indicates that $P$ increases quite rapidly as $m_{q}$ decreases.

To see the $m_{q}$ dependence of the EOS for neutronstar matter, we calculate the mass $(M)$ and the radius $(R)$ of neutron stars for different $m_{q}$ : The TolmanOppenheimer-Volkoff equation is solved by using the EOS of neutron-star matter with neutron, proton, electron and muon under the charge neutrality and beta equilibrium. For asymmetric nuclear matter, we use the parabolic approximation, $\frac{E}{A}(\rho, x)=\frac{E_{Z=N}}{A}(\rho)+(1-$ $2 x)^{2} \epsilon_{\mathrm{sym}}(\rho)$ with the symmetry energy $\epsilon_{\mathrm{sym}}=E_{Z=0} / A-$ $E_{Z=N} / A$ and the proton fraction $x=\rho_{p} / \rho$. This is a standard interpolation between the symmetric nuclear matter and pure neutron matter. The leptons (electrons and muons) are treated as the noninteracting Fermi gas. Solving the Tolman-Oppenheimer-Volkoff equation with a given value of central mass-energy density as an initial condition, we obtain the $M-R$ relation for a spherically symmetric and nonrotating neutron star.

Shown in Fig. 5 is the $M-R$ relation of the neutron star for different $m_{q}$. As $m_{q}$ decreases, the $M-R$ curve 
shifts to the upper right direction, indicating directly the stiffening of our EOS. The maximum mass of the neutron star $\left(M_{\max }\right)$ is found to be 0.53 times the solar mass $\left(M_{\odot}\right)$ at the lightest $m_{q}$ corresponding to $M_{\mathrm{PS}} \simeq 469$ $\mathrm{MeV}$ and $M_{N} \simeq 1161 \mathrm{MeV}$ [20]. Such a maximum mass is too small to account for the observed neutron stars obviously due to the heavy $m_{q}$ : A naive extrapolation of $M_{\max }$ and the corresponding radius to $M_{\mathrm{PS}}=137 \mathrm{MeV}$, with a function $f\left(M_{\mathrm{PS}}\right)=a /\left(M_{\mathrm{PS}}+b\right)+c$, for example, gives $M_{\max }=2.2 M_{\odot}$ and $R=12 \mathrm{~km}$. Although this is a crude estimate, it is encouraging for future quantitative studies [21].

Throughout this Letter, the $N N$ potentials are taken from the zero-strangeness sector of the octet-baryon potentials obtained by lattice QCD simulations with flavor$S U(3)$ symmetry. In this case, the vacuum polarization of the $s$ quark contributes equally to the $u$ and $d$ quarks, though the valence quarks are restricted to only $u$ and $d$ quarks in the $N N$ sector. To be more realistic, we need to consider explicit breaking of flavor-SU(3) symmetry: Studies along this line on the lattice have been already started 12] and results will be implemented in our future EOS calculation with the BHF theory.

To describe the nuclear matter and the neutron matter around the normal nuclear density, it is sufficient to focus on the zero-strangeness sector. However, as the density exceeds a few times the normal nuclear density, hyperons $(Y)$ would start to appear in the ground state, which is particularly relevant to the central core of neutron stars (see e.g. 22] and references therein). Therefore, construction of the EOS with $Y N$ and $Y Y$ interactions is an important next step in our approach. Since the hyperon forces are not well constrained by the experimental data, the results of the lattice QCD simulations [12] are quite useful. The three-baryon interactions [19] with hyperons will also be important to construct a realistic EOS to sustain recently discovered massive neutron stars. The results of the present Letter provide a starting point of all these developments in the near future.

We thank K.-I. Ishikawa and the PACS-CS group for providing their DDHMC/PHMC code [23], and authors and maintainer of CPS ++24 , whose modified version is used in this Letter. Numerical computations of this work have been carried out at Univ. of Tsukuba supercomputer system $(\mathrm{T} 2 \mathrm{~K})$. This research is supported in part by Grant-in-Aid for Scientific Research on Innovative Areas(No.2004:20105001, 20105003) and for Scientific Research (B) 25287046, 24740146, (C) 23540321 and SPIRE (Strategic Program for Innovative REsearch). T.H. was partially supported by RIKEN iTHES Project.

[1] P. Demorest, T. Pennucci, S.M. Ransom, M.S.E. Roberts, and J.W.T. Hessels, Nature (London) 467, 1081
(2010); J. Antoniadis, et al., Science 340, 6131 (2013)

[2] Y. Sekiguchi, K. Kiuchi, K. Kyutoku, and M. Shibata, Phys. Rev. Lett. 107, 051102 (2011)

[3] H.-T. Janka, F. Hanke, L. Huedepohl, A. Marek, B. Mueller, and M. Obergaulinger, Prog. Theor. Phys. 2012, 01A309 (2012)

[4] M. Baldo and G.F. Burgio, Rept. Prog. Phys. 75, 026301 (2012)

[5] J. Carlson, S. Gandolfi, and A. Gezerlis, Prog. Theor. Exp. Phys. 2012, 01A209 (2012)

[6] K. Hebeler, J.M. Lattimer, C.J. Pethick, and A. Schwenk, Astrophys. J. 773, 11 (2013)

[7] J.W. Holt, N. Kaiser and W. Weise, arXiv:1304.6350

[8] T. Inoue, N. Ishii, S. Aoki, T. Dooi, T. Hatsuda, Y. Ikeda, K. Murano H. Nemura, and K. Sasaki (HAL QCD Collaboration), Phys. Rev. Lett. 106, 162002 (2011)

[9] T. Inoue, S. Aoki, T. Doi, T. Hatsuda, Y. Ikeda, N. Ishii, K. Murano, H. Nemura, and K. Sasaki (HAL QCD Collaboration), Nucl. Phys. A 881, 28 (2012)

[10] N. Ishii, S. Aoki, and T. Hatsuda, Phys. Rev. Lett. 99, 022001 (2007) S. Aoki, T. Hatsuda and N. Ishii, Prog. Theor. Phys. 123, 89 (2010)

[11] N. Ishii, S. Aoki, T. Doi, T. Hatsuda, Y. Ikeda, T. Inoue, K. Murano, H. Nemura, and K. Sasaki (HAL QCD Collaboration), Phys. Lett. B712 (2012) 437.

[12] S. Aoki, T. Doi, T. Hatsuda, Y. Ikeda, T. Inoue, N. Ishii, K. Murano, H. Nemura, and K. Sasaki (HAL QCD Collaboration), Prog. Theor. Exp. Phys. 2012, 01A105 (2012)

[13] We note that the absence of the bound deuteron at $M_{\mathrm{PS}} \simeq 469 \mathrm{MeV}$ found in the HAL QCD method is in contrast to the results using the Lüscher's method, although simulation parameters are somewhat different: T. Yamazaki, K.-I. Ishikawa, Y. Kuramashi and A. Ukawa, Phys. Rev. D 86, 074514 (2012) S.R. Beane, E. Chang, W. Detmold, H.W. Lin, T.C. Luu, K. Orginos, A. Parreño, M. J. Savage, A. Torok, and A. Walker-Loud (NPLQCD Collaboration), Phys. Rev. D 85, 054511 (2012)

[14] M.I. Haftel and F. Tabakin, Nucl. Phys. A158, 1 (1970)

[15] H.Q. Song, M. Baldo, G. Giansiracusa, and U. Lombardo, Phys. Rev. Lett. 81, 1584 (1998).

[16] A. Akmal, V.R. Pandharipande and D. G. Ravenhall, Phys. Rev. C 58, 1804 (1998)

[17] M. Baldo, I. Bombaci, L.S. Ferreira, G. Giansiracusa, and U. Lombardo, Phys. Rev. C 43, 2605 (1991).

[18] K. Murano, N. Ishii, S. Aoki, T. Doi, T. Hatsuda, Y. Ikeda, T. Inoue, H. Nemura, and K. Sasaki (HAL QCD Collaboration), arXiv:1305.2293.

[19] T. Doi, S. Aoki, T. Hatsuda, Y. Ikeda, T. Inoue, N. Ishii, K. Murano, H. Nemura, and K. Sasaki (HAL QCD Collaboration), Prog. Theor. Phys. 127, 723 (2012)

[20] $E / A$ and its slope for $M_{\mathrm{PS}}=469 \mathrm{MeV}$ in Fig 4 is already rather close to APR for $k_{F}<1.5 \mathrm{fm}^{-1}$. This implies that small $M$ neutron star with the central density not far from $\rho_{0}$ have a radius of about $10 \mathrm{~km}$. This is actually seen in Fig 5

[21] The crust EOS, which is unknown for unphysical $m_{q}$ and thus is not included in our calculation, would increase $R$ for small $M$ without affecting $M_{\max }$. We checked this qualitatively by matching our EOS with the BaymPethick-Sutherland (BPS) EOS taken from Table 7 of [ [6] .

[22] K. Masuda, T. Hatsuda and T. Takatsuka, Astrophys. J. 764, 12 (2013) 
[23] S. Aoki et al. [PACS-CS Coll.], Phys. Rev. D 79, 034503

http://qcdoc.phys.columbia.edu/cps.html (2009)

[24] Columbia

Physics System

(CPS), 